\title{
Some properties of unitary Cayley graphs
}

\author{
Walter Klotz and Torsten Sander \\ Institut für Mathematik \\ Technische Universität Clausthal, Germany \\ klotz@math.tu-clausthal.de \\ torsten.sander@math.tu-clausthal.de
}

Submitted: Feb 21, 2007; Accepted: May 25, 2007; Published: Jun 21, 2007

Mathematics Subject Classification: 05C25, 05C50

\begin{abstract}
The unitary Cayley graph $X_{n}$ has vertex set $Z_{n}=\{0,1, \ldots, n-1\}$. Vertices $a, b$ are adjacent, if $\operatorname{gcd}(a-b, n)=1$. For $X_{n}$ the chromatic number, the clique number, the independence number, the diameter and the vertex connectivity are determined. We decide on the perfectness of $X_{n}$ and show that all nonzero eigenvalues of $X_{n}$ are integers dividing the value $\varphi(n)$ of the Euler function.
\end{abstract}

\section{Introduction}

Let $\Gamma$ be a multiplicative group with identity 1 . For $S \subseteq \Gamma, 1 \notin S$ and $S^{-1}=\left\{s^{-1}: s \in\right.$ $S\}=S$ the Cayley Graph $X=\operatorname{Cay}(\Gamma, S)$ is the undirected graph having vertex set $V(X)=\Gamma$ and edge set $E(X)=\left\{\{a, b\}: a b^{-1} \in S\right\}$. By right multiplication $\Gamma$ may be considered as a group of automorphisms of $X$ acting transitively on $V(X)$. The Cayley graph $X$ is regular of degree $|S|$. Its connected components are the right cosets of the subgroup generated by $S$. So $X$ is connected, if $S$ generates $\Gamma$. More information about Cayley graphs can be found in the books on algebraic graph theory by Biggs [3] and by Godsil and Royle [10].

For a positive integer $n>1$ the unitary Cayley graph $X_{n}=\operatorname{Cay}\left(Z_{n}, U_{n}\right)$ is defined by the additive group of the ring $Z_{n}$ of integers modulo $n$ and the multiplicative group $U_{n}$ of its units. If we represent the elements of $Z_{n}$ by the integers $0,1, \ldots, n-1$, then it is well known [13] that

$$
U_{n}=\left\{a \in Z_{n}: \operatorname{gcd}(a, n)=1\right\} .
$$

So $X_{n}$ has vertex set $V\left(X_{n}\right)=Z_{n}=\{0,1, \ldots, n-1\}$ and edge set

$$
E\left(X_{n}\right)=\left\{\{a, b\}: a, b \in Z_{n}, \operatorname{gcd}(a-b, n)=1\right\} .
$$

The graph $X_{n}$ is regular of degree $\left|U_{n}\right|=\varphi(n)$, where $\varphi(n)$ denotes the Euler function. If $n=p$ is a prime number, then $X_{n}=K_{p}$ is the complete graph on $p$ vertices. If $n=p^{\alpha}$ is a 
prime power then $X_{n}$ is a complete $p$-partite graph which has the residue classes modulo $p$ in $Z_{n}$ as maximal sets of independent (pairwise nonadjacent) vertices. Unitary Cayley graphs are highly symmetric. They have some remarkable properties connecting graph theory and number theory.

In some recent papers induced cycles in $X_{n}$ were investigated. Berrizbeitia and Giudici [2] studied the number $p_{k}(n)$ of induced $k$-cycles in $X_{n}$. Fuchs and Sinz [8, 9] showed that the maximal length of an induced cycle in $X_{n}$ is $2^{r}+2$, where $r$ is the number of different prime divisors of $n$.

In Section 2 we deal with some basic invariants of $X_{n}$. We show that the chromatic number $\chi\left(X_{n}\right)$ and the clique number $\omega\left(X_{n}\right)$ equal the smallest prime divisor $p$ of $n$. For the complementary graph $\bar{X}_{n}$ of $X_{n}$ we have $\chi\left(\bar{X}_{n}\right)=\omega\left(\bar{X}_{n}\right)=n / p$. Unitary Cayley graphs represent very reliable networks, which means that the vertex connectivity $\kappa\left(X_{n}\right)$ equals the degree of regularity of $X_{n}, \kappa\left(X_{n}\right)=\varphi(n)$. We show that the diameter of $X_{n}$ is at most 3 .

A graph $G$ is perfect, if for every induced subgraph $G^{\prime} \subseteq G$ chromatic number and clique number coincide, $\chi\left(G^{\prime}\right)=\omega\left(G^{\prime}\right)$. In Section 3 we prove that $X_{n}$ is perfect, if and only if $n$ is even or if $n$ is odd and has at most two different prime divisors.

The eigenvalues of a graph $G$ are the eigenvalues of an arbitrary adjacency matrix of $G$. In Section 4 we show that all nonzero eigenvalues of $X_{n}$ are divisors of $\varphi(n)$. The definition of $X_{n}$ is extended to gcd-graphs $X_{n}(D)$, where vertices $a, b$ are adjacent, if $\operatorname{gcd}(a-b, n) \in D, D$ a given set of divisors of $n$. All eigenvalues of $X_{n}(D)$ also turn out to be integral.

\section{Basic invariants}

First we determine the chromatic number and the clique number of $X_{n}$ and of the complementary graph $\bar{X}_{n}$. We remark that $\omega\left(\bar{X}_{n}\right)$ and $\chi\left(\bar{X}_{n}\right)$ are also called the independence number and the clique covering number of $X_{n}$. From now on we always assume that $n$ is an integer, $n \geq 2$.

Theorem 1. If $p$ is the smallest prime divisor of $n$, then we have

$$
\chi\left(X_{n}\right)=\omega\left(X_{n}\right)=p, \quad \chi\left(\bar{X}_{n}\right)=\omega\left(\bar{X}_{n}\right)=\frac{n}{p} .
$$

Proof. As the vertices $0,1, \ldots, p-1$ induce a clique in $X_{n}$, we have

$$
\chi\left(X_{n}\right) \geq \omega\left(X_{n}\right) \geq p .
$$

On the other hand the residue classes modulo $p$ in $Z_{n}=\{0,1, \ldots, n-1\}$ constitute $p$ sets of independent vertices of $X_{n}$. These sets can be taken as color classes to show $\chi\left(X_{n}\right) \leq p$, which proves equality in (1). In $\bar{X}_{n}$ the residue classes induce cliques showing

$$
\chi\left(\bar{X}_{n}\right) \geq \omega\left(\bar{X}_{n}\right) \geq \frac{n}{p} .
$$


Now the integer intervals

$$
\{k p, k p+1, \ldots,(k+1) p-1\}, \quad 0 \leq k \leq \frac{n}{p}-1,
$$

consist of indpendent vertices of $\bar{X}_{n}$. These sets can be taken as color classes for $\bar{X}_{n}$ to establish $\chi\left(\bar{X}_{n}\right) \leq n / p$, which proves equality in (2).

Corollary 2. [7] The unitary Cayley graph $X_{n}, n \geq 2$, is bipartite if and only if $n$ is even.

Corollary 3. There is no selfcomplementary unitary Caley graph $X_{n}$ for $n \geq 2$.

Proof. Suppose $X_{n}$ is selfcomplementary. Then $X_{n}$ and $\bar{X}_{n}$ must have the same chromatic number, which by Theorem 1 implies $n=p^{2}, p$ a prime. As $X_{n} \cup \bar{X}_{n}=K_{n}$ is the complete graph on $n$ vertices and as $X_{n}$ and $\bar{X}_{n}$ must have the same degree of regularity, we conclude

$$
\varphi(n)=\frac{n-1}{2} .
$$

Inserting $n=p^{2}$ we get $2 \varphi\left(p^{2}\right)=2 p(p-1)=p^{2}-1$, which is impossible.

We remark that Corollary 3 supports a still open conjecture of Lehmer [12], which states that equation (3) is unsolvable.

Let $a \in U_{n}$, i.e. $1 \leq a \leq n-1, \operatorname{gcd}(a, n)=1$. If $n \geq 3$, then the sequence

$$
\left(x_{k}\right), x_{k} \equiv k a \bmod n, 0 \leq k \leq n-1 \text {, }
$$

defines a hamiltonian cycle $C_{a}$ of $X_{n}$. We notice that $a$ and $n-a$ define the same hamiltonian cycle. There are $\varphi(n) / 2$ edge disjoint hamiltonian cycles $C_{a}, a \in U_{n}$, which completely partition the edge set $E\left(X_{n}\right)$. This implies that $X_{n}$ has edge connectivity $\varphi(n)$. We show that this is also the value of the vertex connectivity of $X_{n}$.

Theorem 4. The unitary Cayley graph $X_{n}$ has vertex connectivity $\kappa(n)=\varphi(n)$.

Proof. For $a \in Z_{n}$ and $b \in Z_{n}$ we define the affine transformation

$$
\psi_{a, b}: Z_{n} \longrightarrow Z_{n} \text { by } \psi_{a, b}(x) \equiv a x+b \bmod n \text { for } x \in Z_{n} .
$$

We check that $\psi_{a, b}$ is an automorphism of $X_{n}$, if and only if $a \in U_{n}$. Moreover, $A\left(X_{n}\right)=$ $\left\{\psi_{a, b}: a \in U_{n}, b \in Z_{n}\right\}$ is a subgroup of the automorphism group $\operatorname{Aut}\left(X_{n}\right)$. We call $A\left(X_{n}\right)$ the group of affine automorphisms of $X_{n}$.

According to Biggs [3] a graph $G$ is called symmetric, if for all vertices $x, y, u, v$ of $G$ such that $x$ is adjacent to $y$ and $u$ is adjacent to $v$, there is an automorphism $\sigma$ of $G$ for which $\sigma(x)=u$ and $\sigma(y)=v$. If $G=X_{n}$, then we find exactly one automorphism $\sigma \in A\left(X_{n}\right)$ satisfying these conditions. So $X_{n}$ is symmetric.

It has been shown by Watkins [15], see also [4], that the vertex connectivity $\kappa(G)$ of a connected graph $G$, which is regular and edge transitive, equals its degree of regularity. This result especially applies to connected, symmetric graphs, because symmetry includes regularity and edge transitivity [3]. Therefore, we conclude $\kappa\left(X_{n}\right)=\varphi(n)$. 
The following lemma will be used to determine the number of common neighbors of a pair of vertices in $X_{n}$.

Lemma 5. For integers $n, s, n \geq 2$, denote by $F_{n}(s)$ the number of solutions of the congruence

$$
x+y \equiv s \bmod n, x \in U_{n}, y \in U_{n} .
$$

Then we have

$$
F_{n}(s)=n \prod_{p \mid n, p \text { prime }}\left(1-\frac{\varepsilon(p)}{p}\right), \text { where } \varepsilon(p)=\left\{\begin{array}{ll}
1, & \text { if } p \mid s \\
2, & \text { if } p \nmid s
\end{array} .\right.
$$

Proof. Let $p_{1}, p_{2}, \ldots, p_{r}$ be the different prime divisors of $n$,

$$
n=p_{1}^{\alpha_{1}} p_{2}^{\alpha_{2}} \cdots p_{r}^{\alpha_{r}}, m=p_{1} p_{2} \cdots p_{r} .
$$

If $x$ and $y$ satisfy (4), then $y$ is uniquely determined modulo $n$ by $x$. So we have to find out the number of possible entries for $x$. We partition the interval of integers $[0, n-1]=$ $\{0,1, \ldots, n-1\}$ into the disjoint intervals $I_{k}=[(k-1) m, k m-1], k=1, \ldots, n / m$. By the Chinese Remainder Theorem [13] every integer $x \in I_{k}$ is uniquely determined by its values $x_{i}$ modulo $p_{i}, 1 \leq i \leq r$, i.e. by the congruences

$$
x \equiv x_{i} \bmod p_{i}, 0 \leq x_{i} \leq p_{i}-1,1 \leq i \leq r .
$$

To guarantee $x \in I_{k} \cap U_{n}$ all $x_{i}$ must be nonzero. There remain $p_{i}-1$ possible choices for $x_{i}$. An additional value for $x_{i}$ has to be ruled out, if $s \equiv s^{\prime} \bmod p_{i}, 0<s^{\prime}<p_{i}$. In this case $x_{i}=s^{\prime}$ would have the consequence $y \equiv 0 \bmod p_{i}$ implying $y \notin U_{n}$. So the number of possible choices for $x \in I_{k} \cap U_{n}$ to satisfy (4) is $\left(p_{1}-\varepsilon\left(p_{1}\right)\right) \cdots\left(p_{r}-\varepsilon\left(p_{r}\right)\right)$. Multiplying this number by the number $n / m$ of intervals $I_{k}$ proves Lemma 5 .

Theorem 6. In the notation of Lemma 5 the number of common neighbors of distinct vertices $a, b$ in the unitary Cayley graph $X_{n}$ is given by $F_{n}(a-b)$.

Proof. Let $a, b, z$ be elements of $V\left(X_{n}\right)=Z_{n}=\{0,1, \ldots, n-1\}$. Vertex $z$ is a common neighbor of $a$ and $b$, if and only if $\operatorname{gcd}(a-z, n)=\operatorname{gcd}(z-b, n)=1$. There exist unique $x, y \in Z_{n}$ such that

$$
a-z \equiv x \bmod n, z-b \equiv y \bmod n .
$$

Now $z \equiv a-x \equiv b+y$ becomes a common neighbor of $a$ and $b$, if and only if

$$
x+y \equiv a-b \bmod n, x \in U_{n}, y \in U_{n} .
$$

By Lemma 5 this means that the number of common neighbors of $a$ and $b$ is $F_{n}(a-b)$.

Corollary 7. Every pair of adjacent vertices of $X_{n}$ has the same number $\lambda(n)$ of common neighbors,

$$
\lambda(n)=n \prod_{p \mid n, p \text { prime }}\left(1-\frac{2}{p}\right) .
$$


Corollary 8. [7] The number $T(n)$ of triangles in $X_{n}$ is

$$
T(n)=\frac{n^{3}}{6} \prod_{p \mid n, p \text { prime }}\left(1-\frac{1}{p}\right)\left(1-\frac{2}{p}\right) .
$$

Proof. By Corollary 7 every edge of $X_{n}$ is contained in $\lambda(n)$ triangles. If we multiply $\lambda(n)$ by the number $n \varphi(n) / 2$ of edges in $X_{n}$, then every triangle is counted three times. So we have $T(n)=n \varphi(n) \lambda(n) / 6$. The result follows, if we insert $\lambda(n)$ from Corollary 7 and the analogous product expansion for $\varphi(n)$ [13].

The distance $\mathrm{d}(x, y)$ of vertices $x$ and $y$ of a graph $G$ is the length (number of edges) of a shortest $x, y$-path. The diameter $\operatorname{diam}(G)$ is the maximal distance any two vertices of $G$ may have.

Theorem 9. For $n \geq 2$ we have

$$
\operatorname{diam}\left(X_{n}\right)=\left\{\begin{array}{l}
1, \text { if } n \text { is a prime number } \\
2, \text { if } n=2^{\alpha}, \alpha>1, \\
2, \text { if } n \text { is odd, but not a prime number, } \\
3, \text { if } n \text { is even and has an odd prime divisor }
\end{array}\right.
$$

Proof. If $n$ is a prime number, then $X_{n}=K_{n}$ is the complete graph, which has diameter 1. In the other cases $X_{n}$ is not complete, $\operatorname{diam}\left(X_{n}\right) \geq 2$. If $n=2^{\alpha}, \alpha>1$, then $X_{n}$ is the complete bipartite graph with vertex partition $V\left(X_{n}\right)=\{0,2, \ldots, n-2\} \cup\{1,3, \ldots, n-1\}$, which has diameter 2 .

Suppose $n$ is odd, but not a prime number. By Theorem 6 the number of common neighbors of vertices $a \neq b$ is $F_{n}(a-b)$. According to Lemma 5 all factors in the expansion of $F_{n}(a-b)$ are positive, if $n$ has only odd prime divisors. In this case there is a common neighbor to every pair of distinct vertices, which implies $\operatorname{diam}\left(X_{n}\right)=2$.

Finally, we consider the case where $n$ is even and has an odd prime divisor $p$. The vertices 0 and $p$ of $X_{n}$ are not adjacent and by Theorem 6 they have no common neighbor. Therefore, we have $\operatorname{diam}\left(X_{n}\right) \geq \mathrm{d}(0, p) \geq 3$. Suppose now that $a$ and $b, a \neq b$, are arbitrary nonadjacent vertices of $X_{n}$, which have no common neighbor. Any two vertices $x$ and $y, x \neq y$, of $X_{n}$, which are both even or both odd have a common neighbor by Theorem 6 . So we may assume that $a$ is even and $b$ is odd. All $\varphi(n)$ neighbors of $a$ are odd. Let $c$ be one of them. Now $c$ and $b$ are both odd and therefore have a common neighbor $d$. Passing along $a, c, d, b$ shows $\mathrm{d}(a, b) \leq 3, \operatorname{diam}\left(X_{n}\right)=3$.

\section{$3 \quad$ Perfectness}

A graph $G$ is perfect [1], if for every induced subgraph $G^{\prime} \subseteq G$ the clique number and the chromatic number coincide, $\omega\left(G^{\prime}\right)=\chi\left(G^{\prime}\right)$. Clearly, induced cycles of odd length at least 5, popularly called odd holes, prevent a graph from being perfect. Chudnovsky, Robertson, Seymour, and Thomas [5] in 2002 turned the corresponding famous conjecture of Berge into the following theorem, the Strong Perfect Graph Theorem (SPGT). 
SPGT. A graph $G$ is perfect if and only if $G$ and its complement $\bar{G}$ have no odd holes.

If $n$ is even or a power of a prime $p$, then $X_{n}$ is bipartite or completely $p$-partite. As these graphs are perfect, we may assume that $n$ is odd and has at least two different prime divisors.

Lemma 10. If $n$ is odd and has at least three different prime divisors, then $X_{n}$ contains an induced cycle $C_{5}$ of length 5.

Proof. Let $p_{1}, \ldots, p_{r}$ (in ascending order) be the prime divisors of the odd integer $n, r \geq$ $3, m=p_{1} p_{2} \cdots p_{r}$. As the cycle $C_{5}$ is selfcomplementary, it suffices to show that there is an induced $C_{5}$ in the complement $\bar{X}_{n}$. We define the vertices $x_{0}, x_{1}, x_{2}, x_{3}$ by

$$
x_{0}=0, x_{1}=p_{r}, x_{2}=p_{r}+p_{1} \cdots p_{r-1}, x_{3}=2 p_{r}+p_{1} \cdots p_{r-1} \text {. }
$$

According to the Chinese Remainder Theorem we can define $x_{4} \in Z_{m}$ uniquely by the following congruences.

$$
\begin{aligned}
& x_{4} \equiv 0 \bmod p_{1}, x_{4} \equiv 2 p_{r} \bmod p_{2}, x_{4} \equiv 2 p_{1} \cdots p_{r-1} \bmod p_{r}, \\
& x_{4} \equiv 0 \bmod p_{j} \text { for } j=3, \ldots, r-1
\end{aligned}
$$

One checks that the vertices $x_{0}, \ldots, x_{4}$ are distinct. They define a cycle $C_{5}$ of $\bar{X}_{n}$, because

$$
\begin{aligned}
& x_{1}-x_{0} \equiv x_{3}-x_{2} \equiv 0 \bmod p_{r}, \\
& x_{2}-x_{1} \equiv x_{4}-x_{0} \equiv 0 \bmod p_{1}, x_{4}-x_{3} \equiv 0 \bmod p_{2}
\end{aligned}
$$

imply that the edges $\left\{x_{0}, x_{1}\right\}, \ldots,\left\{x_{4}, x_{0}\right\}$ belong to $\bar{X}_{n}$. It remains to show that this $C_{5}$ has no chords in $\bar{X}_{n}$.

We have $x_{2}-x_{0}=p_{r}+p_{1} \cdots p_{r-1}$, which implies $\operatorname{gcd}\left(x_{2}-x_{0}, m\right)=1$ and $\left\{x_{0}, x_{2}\right\} \notin$ $E\left(\bar{X}_{n}\right)$. A similar argument applies to the edges $\left\{x_{0}, x_{3}\right\}$ and $\left\{x_{1}, x_{3}\right\}$. Consider now the edge $\left\{x_{1}, x_{4}\right\}$. By (5) and (6) we conclude that

$$
\begin{aligned}
& x_{4}-x_{1} \equiv-p_{r} \bmod p_{1}, \text { which implies } p_{1} \backslash\left(x_{4}-x_{1}\right), \\
& x_{4}-x_{1} \equiv p_{r} \bmod p_{2}, \text { which implies } p_{2} \backslash\left(x_{4}-x_{1}\right), \\
& x_{4}-x_{1} \equiv-p_{r} \bmod p_{j}, j=3, \ldots, r-1, \text { which implies } p_{j} \backslash\left(x_{4}-x_{1}\right), \\
& x_{4}-x_{1} \equiv 2 p_{1} \cdots p_{r-1} \bmod p_{r}, \text { which implies } p_{r} \backslash\left(x_{4}-x_{1}\right) .
\end{aligned}
$$

Now $\operatorname{gcd}\left(x_{4}-x_{1}, m\right)=1$ implies that the edge $\left\{x_{1}, x_{4}\right\}$ does not belong to $\bar{X}_{n}$. Similarly, we confirm $\left\{x_{2}, x_{4}\right\} \notin E\left(\bar{X}_{n}\right)$.

It has been shown by Fuchs and Sinz $[8,9]$ that the length of a longest induced cycle in $X_{n}$ is $2^{r}+2$, if $r \geq 2$ is the number of distinct prime divisors of $n$. We remark that their arguments can also be used to show that the length of a longest induced path in $X_{n}$ is $2^{r}$.

To complete our decision concerning the perfectness of $X_{n}$, it remains to investigate the case where $n$ has exactly two different odd prime divisors. By the just mentioned result we know that a longest induced cycle in $X_{n}$ has length 6 . So the only possible odd hole $X_{n}$ may have is $C_{5}$. But this is excluded by the next lemma. 
Lemma 11. If $n$ is odd and has exactly two different prime divisors, then $\bar{X}_{n}$ has no odd hole $C_{2 k+1}, k \geq 2$.

Proof. Assume that $\bar{X}_{n}$ contains the induced cycle $C_{2 k+1}, k \geq 2$, which runs through the vertices $x_{0}, x_{1}, \ldots, x_{2 k}$ in this order. If $p_{1}, p_{2}$ are the two odd prime divisors of $n$, then for every edge $\left\{x_{j}, x_{j+1}\right\}$ (indices modulo $\left.2 k+1\right)$ we must have $p_{1} \mid\left(x_{j+1}-x_{j}\right)$ or $p_{2} \mid\left(x_{j+1}-x_{j}\right)$. For every pair of consecutive edges $\left\{x_{j}, x_{j+1}\right\},\left\{x_{j+1}, x_{j+2}\right\}$ of $C_{2 k+1}$ the prime divisors $p_{1}, p_{2}$ must alternate. Otherwise we would have e.g. $x_{j}-x_{j+1}=$ $s p_{1}, x_{j+2}-x_{j+1}=t p_{1}$, which would imply $x_{j+2}-x_{j}=(t-s) p_{1}$. But this would mean that $\left\{x_{j}, x_{j+2}\right\} \in E\left(\bar{X}_{n}\right)$ is a chord of $C_{2 k+1}$ contradicting our asumption. On the other hand the alternation of prime divisors along the edges of $C_{2 k+1}$ forces the cycle to have even length, which again is a contradiction.

With the help of SPGT, Lemma 10 and Lemma 11 we have established the following theorem.

Theorem 12. The unitary Cayley graph $X_{n}, n \geq 2$, is perfect if and only if $n$ is even or if $n$ is odd and has at most two different prime divisors.

\section{Eigenvalues}

The eigenvalues of a graph $G$ are the eigenvalues of an arbitrary adjacency matrix of $G$. We establish the adjacency matrix $A_{n}$ of $X_{n}$ with respect to the natural order of the vertices $0,1, \ldots, n-1$. The entries $a_{0}, a_{1}, \ldots, a_{n-1}$ of the first row of $A_{n}$ generate the entries of the other rows by a cyclic shift.

$$
A_{n}=\left(\begin{array}{llll}
a_{0} & a_{1} & \ldots & a_{n-1} \\
a_{n-1} & a_{0} & \ldots & a_{n-2} \\
\cdot & \cdot & \ldots & \cdot \\
\cdot & \cdot & \ldots & \cdot \\
a_{1} & a_{2} & \ldots & a_{0}
\end{array}\right)
$$

Matrices of this kind are called circulant matrices. A circulant graph is a graph, which has a circulant adjacency matrix. Circulant graphs with $n$ vertices are exactly the graphs isomorphic to a Cayley graph with respect to the additive group $Z_{n}$ of integers modulo $n$. Unitary Cayley graphs are circulant graphs.

A detailed exposition of circulant matrices is given by Davis [6]. There is an explicit formula for the eigenvalues $\lambda_{r}, 0 \leq r \leq n-1$, of a circulant matrix such as $A_{n}$. Define the polynomial $p_{n}(z)$ by the entries of the first row of $A_{n}$ and let $w$ denote a complex primitive $n$-th root of unity.

$$
p_{n}(z)=\sum_{j=0}^{n-1} a_{j} z^{j}, \quad w=\exp \left(\frac{2 \pi i}{n}\right)
$$


The eigenvalues of $A_{n}$ are given by (cf. Theorem 3.2.2 [6])

$$
\lambda_{r}=p_{n}\left(w^{r}\right), 0 \leq r \leq n-1 .
$$

Here every eigenvalue of $A_{n}$ is listed according to its multiplicity. The definition of $A_{n}$ as a special adjacency matrix of $X_{n}$ implies

$$
a_{j}=\left\{\begin{array}{l}
1, \text { if } \operatorname{gcd}(j, n)=1 \\
0, \text { if } \operatorname{gcd}(j, n)>1
\end{array}\right.
$$

Therefore, equation (7) leads to

$$
\lambda_{r}=\sum_{\substack{1 \leq j<n \\ \operatorname{gcd}(j, n)=1}} w^{r j}=c(r, n), 0 \leq r \leq n-1 .
$$

The arithmetic function $c(r, n)$ is a Ramanujan sum, for which some results are available [14]. For integers $r, n, n>0$, Ramanujan sums have only integral values. So all eigenvalues of unitary Cayley graphs are integers. More information can be drawn from the following formula (cf. Corollary 2.4 of [14]).

$$
\lambda_{r}=c(r, n)=\mu\left(t_{r}\right) \frac{\varphi(n)}{\varphi\left(t_{r}\right)}, \text { where } t_{r}=\frac{n}{\operatorname{gcd}(r, n)}, 0 \leq r \leq n-1 .
$$

Here $\mu$ denotes the Möbius function.

Theorem 13. For $n \geq 2$, the following statements hold.

1. Every nonzero eigenvalue of $X_{n}$ is a divisor of $\varphi(n)$.

2. Let $m$ be the maximal squarefree divisor of $n$. Then

$$
\lambda_{\min }=\mu(m) \frac{\varphi(n)}{\varphi(m)}
$$

is a nonzero eigenvalue of $X_{n}$ of minimal absolute value and multiplicity $\varphi(m)$. Every eigenvalue of $X_{n}$ is a multiple of $\lambda_{\min }$. If $n$ is odd, then $\lambda_{\min }$ is the only nonzero eigenvalue of $X_{n}$ with minimal absolute value. If $n$ is even, then $-\lambda_{\min }$ is also an eigenvalue of $X_{n}$ with multiplicity $\varphi(m)$.

Proof. 1. By the multiplicative properties of the Euler function $\varphi(t)$ divides $\varphi(n)$, if $t$ is a divisor of $n$ [13]. Therefore, (9) implies that the nonzero eigenvalues of $X_{n}$ are divisors of $\varphi(n)$.

2. For $\lambda_{r} \neq 0$ we must have $\mu\left(t_{r}\right) \neq 0$, which is equivalent to $t_{r}$ being a divisor of $m$. Now by (9) the absolute value of $\lambda_{r} \neq 0$ is minimal if and only if $\varphi\left(t_{r}\right)=\varphi(m)$. This equation always has the trivial solution $t_{r}=m$, which implies

$$
\lambda_{r}=\lambda_{\min }=\mu(m) \frac{\varphi(n)}{\varphi(m)} .
$$


For $0 \leq r \leq n-1$ we have the equivalences

$$
\begin{gathered}
\lambda_{r}=\lambda_{\text {min }} \Longleftrightarrow t_{r}=\frac{n}{\operatorname{gcd}(r, n)}=m \Longleftrightarrow \operatorname{gcd}(r, n)=\frac{n}{m} \\
\Longleftrightarrow r \in Q:=\left\{x \frac{n}{m}: 0 \leq x<m, \operatorname{gcd}(x, m)=1\right\} .
\end{gathered}
$$

So $\lambda_{\text {min }}$ has multiplicity $|Q|=\varphi(m)$.

If $\lambda_{r}$ is an arbitrary nonzero eigenvalue of $X_{n}$, then $t_{r}$ is a divisor of $m$ and so $\varphi\left(t_{r}\right)$ divides $\varphi(m)$, say $\varphi(m)=k \varphi\left(t_{r}\right)$ with a positive integer $k$. Now $\lambda_{r}$ becomes a multiple of $\lambda_{\min }$ by (9) and (10),

$$
\lambda_{r}=\mu\left(t_{r}\right) \frac{\varphi(n)}{\varphi\left(t_{r}\right)}=k \mu\left(t_{r}\right) \frac{\varphi(n)}{\varphi(m)}= \pm k \lambda_{\min } .
$$

If $n$ is odd and $t_{r}$ divides $m$, then $\varphi\left(t_{r}\right)=\varphi(m)$ has only the trivial solution $t_{r}=m$ and $\lambda_{\min }$ is the only eigenvalue with minimal absolute value. But if $n$ is even, we have $\varphi(m)=\varphi(m / 2)$ and we get another solution for $t_{r}=m / 2$,

$$
\lambda_{\text {min }}^{\prime}=\mu\left(\frac{m}{2}\right) \frac{\varphi(n)}{\varphi\left(\frac{m}{2}\right)}=-\mu(m) \frac{\varphi(n)}{\varphi(m)}=-\lambda_{\min } .
$$

As above we deduce the multiplicity $\varphi(m / 2)=\varphi(m)$ for the eigenvalue $\lambda_{m i n}^{\prime}$.

Remember that $X_{n}$ is bipartite for even $n$. The appearence of $\lambda_{\min }$ and $-\lambda_{\min }$ in this case reflects the well known fact [3] that the nonzero eigenvalues of bipartite graphs occur in pairs $(\lambda,-\lambda)$ with the same multiplicity. We further mention that for the connected graph $X_{n}$ the degree of regularity, i.e. $\varphi(n)$, is a simple eigenvalue of maximal absolute value.

Corollary 14. There is an eigenvalue \pm 1 of $X_{n}$, if and only if $n$ is squarefree. If $n$ is squarefree, then $X_{n}$ has the eigenvalue $\mu(n)$ with multiplicity $\varphi(n)$. The unitary Cayley graph $X_{n}$ has both eigenvalues \pm 1 with multiplicity $\varphi(n)$, if and only if $n$ is squarefree and even.

Theorem 15. Let $m$ be the maximal squarefree divisor of $n$ and let $M$ be the set of positive divisors of $m$. Then the following statements for the unitary Cayley graph $X_{n}, n \geq 2$, hold.

1. Repeating every term of the sequence

$$
S=\left(\mu(t) \frac{\varphi(n)}{\varphi(t)}\right)_{t \in M}
$$

$\varphi(t)$-times results in a sequence $\tilde{S}$ of length $m$ which consists of all nonzero eigenvalues of $X_{n}$ such that the number of appearences of an eigenvalue is its multiplicity. 
2. The multiplicity of zero as an eigenvalue of $X_{n}$ is $n-m$.

3. If $\alpha(\lambda)$ is the multiplicity of the eigenvalue $\lambda$ of $X_{n}$, then $\lambda \alpha(\lambda)$ is a multiple of $\varphi(n)$.

Proof. 1. The number of terms in the resulting sequence $\tilde{S}$ is

$$
\sum_{t \in M} \varphi(t)=\sum_{t \mid m} \varphi(t)=m
$$

The last equation exhibits the well known summatory function of the Euler function [13].

Equation (9) describes the sequence $\left(\lambda_{r}\right), 0 \leq r \leq n-1$, of all eigenvalues of $X_{n}$, in which each eigenvalue is listed according to its multiplicity. As $\mu\left(t_{r}\right)=0$ for $t_{r} \notin M$, we get the subsequence $\tilde{T}$ of nonzero eigenvalues for $0 \leq r \leq n-1, t_{r} \in M$.

$$
\tilde{T}=\left(\mu\left(t_{r}\right) \frac{\varphi(n)}{\varphi\left(t_{r}\right)}\right)_{0 \leq r \leq n-1, t_{r} \in M}, \quad t_{r}=\frac{n}{\operatorname{gcd}(r, n)}
$$

Let $t$ be an arbitrary element of $M$. For $0 \leq r \leq n-1$, i.e. $r \in Z_{n}$, we have $t_{r}=t$, if and only if $\operatorname{gcd}(r, n)=n / t$. Elementary number theory shows

$$
Q_{t}:=\left\{r \in Z_{n}: \operatorname{gcd}(r, n)=\frac{n}{t}\right\}=\left\{x \frac{n}{t}: x \in Z_{t}, \operatorname{gcd}(x, t)=1\right\},
$$

which implies that $Q_{t}$ has $\varphi(t)$ elements. Therefore, the sequence $\tilde{T}$ consists of all terms

$$
\mu(t) \frac{\varphi(n)}{\varphi(t)}, \quad t \in M
$$

where each of these terms appears $\varphi(t)$-times. If we take every term only once, then we arrive at the sequence $S$ and see that $\tilde{S}$ and $\tilde{T}$ coincide apart possibly from the order of their elements.

2. By (1.) the length of the sequence $\tilde{S}$ equals the number of nonzero eigenvalues, each of them counted according to its multiplicity. As $\tilde{S}$ has length $m$, the eigenvalue zero has multiplicity $n-m$.

3. The statement is trivially true for $\lambda=0$. Let $\lambda$ be a nonzero eigenvalue of $X_{n}$. Then there is an integer $t \in M$ such that $\lambda=\mu(t) \varphi(n) / \varphi(t)$. By (1.) $\lambda$ has at least multiplicity $\varphi(t)$, more precisely

$$
\alpha(\lambda)=k_{t} \varphi(t), \quad k_{t}=|\{\tau \in M: \mu(\tau)=\mu(t), \varphi(\tau)=\varphi(t)\}| .
$$

Now we deduce

$$
\lambda \alpha(\lambda)=\mu(t) \frac{\varphi(n)}{\varphi(t)} k_{t} \varphi(t)=\mu(t) k_{t} \varphi(n) .
$$


We extend the class of unitary Cayley graphs. Let $D$ be a set of positive, proper divisors of the integer $n>1$. Define the gcd-graph $X_{n}(D)$ to have vertex set $Z_{n}=$ $\{0,1, \ldots, n-1\}$ and edge set

$$
E\left(X_{n}(D)\right)=\left\{\{a, b\}: a, b \in Z_{n}, \operatorname{gcd}(a-b, n) \in D\right\} .
$$

If $D=\left\{d_{1}, \ldots, d_{k}\right\}$, then we also write $X_{n}(D)=X_{n}\left(d_{1}, \ldots, d_{k}\right)$, especially $X_{n}(1)=X_{n}$.

Theorem 16. All eigenvalues of gcd-graphs are integers.

Proof. As $X_{n}(D)$ is a circulant graph, its eigenvalues are determined by (7),

$$
\begin{gathered}
\lambda_{r}=\sum_{j=0}^{n-1} a_{j} w^{r j}, \quad 0 \leq r \leq n-1, \text { where } \\
a_{j}=\left\{\begin{array}{l}
1, \text { if } \operatorname{gcd}(j, n) \in D \\
0, \text { if } \operatorname{gcd}(j, n) \notin D . \quad w=\exp \left(\frac{2 \pi i}{n}\right) .
\end{array}\right.
\end{gathered}
$$

Inserting $a_{j}$ yields

$$
\lambda_{r}=\sum_{d \in D} \sum_{\substack{1 \leq j<n \\ \operatorname{gcd}(j, n)=d}} w^{r j}, \quad 0 \leq r \leq n-1 .
$$

Observing

$$
\{j: 1 \leq j<n, \operatorname{gcd}(j, n)=d\}=\{t d: 1 \leq t<n / d, \operatorname{gcd}(t, n / d)=1\}
$$

leads to

$$
\begin{gathered}
\lambda_{r}=\sum_{d \in D} \sum_{\substack{1 \leq t<n / d \\
\operatorname{gcd}(t, n / d)=1}} w^{r t d}=\sum_{d \in D} \sum_{\substack{1 \leq t<n / d \\
\operatorname{gcd}(t, n / d)=1}} \exp \left(\frac{2 \pi i}{n / d} r t\right) \\
\lambda_{r}=\sum_{d \in D} c(r, n / d), 0 \leq r \leq n-1 .
\end{gathered}
$$

Similar to (8) we get a representation of the eigenvalues by Ramanujan sums. As these sums are integer valued, we conclude that all eigenvalues of gcd-graphs are integers.

\section{$5 \quad$ Problems and remarks}

1. Investigate the automorphism group $\operatorname{Aut}\left(X_{n}\right)$. For $n>6$ it seems to be considerably larger than the group $A\left(X_{n}\right)$ of affine automorphisms.

2. Which circulant graphs have only integer eigenvalues? For general considerations about graphs with integral spectra see [11].

3. Investigate the gcd-graphs defined in Section 4 . The graph $X_{n}\left(d_{1}, \ldots, d_{k}\right)$ is connected, if and only if $\operatorname{gcd}\left(d_{1}, \ldots, d_{k}\right)=1$. Examples suggest that chromatic number and clique number of $X_{n}(D)$ are always divisors of $n$.

Acknowledgement. We would like to thank our colleague J. W. Sander for some support in number theory. 


\section{References}

[1] Alfonsin, J. L. R., And Reed, B. A. Perfect Graphs. John Wiley \& Sons, New York-Chichester-Brisbane, 2001.

[2] Berrizbeitia, P., And Giudici, R. E. On cycles in the sequence of unitary Cayley graphs. Discrete Math. 282, 1-3 (2004), 239-243.

[3] Biggs, N. Algebraic graph theory. Second Edition. Cambridge Mathematical Library. Cambridge University Press, 1993.

[4] Boesch, F., And Tindell, R. Circulants and their connectivities. J. Graph Theory 8 (1984), 487-499.

[5] Chudnovsky, Robertson, Seymour, Thomas. The strong perfect graph theorem. Annals of Math. 164, (2002), 51-229.

[6] Davis, P. J. Circulant matrices. A Wiley-Interscience Publication, Pure and Applied Mathematics. John Wiley \& Sons, New York-Chichester-Brisbane, 1979.

[7] Dejter, I. J., And Giudici, R. E. On unitary Cayley graphs. J. Combin. Math. Combin. Comput. 18 (1995), 121-124.

[8] Fuchs E. D. Longest induced cycles in circulant graphs. The Electronic J. Comb. 12 (2005), 1-12.

[9] Fuchs E. D., Sinz J. Longest induced cycles in Cayley graphs. eprint arXiv:math/0410308 (2004), 1-16.

[10] Godsil, C., And Royle, G. Algebraic graph theory. Graduate Texts in Mathematics. Vol 207. Springer, 2001.

[11] Harary, F., And Schwenk, A. J. Which graphs have integral spectra? Lecture Notes in Mathematics 406, Springer Verlag (1974), 45-50.

[12] Lehmer D. H. On Euler's totient function. Bull. Amer. Math. Soc. 38 (1932), 745-751.

[13] Rose H. E. A course in number theory. Oxford Science Publications. Oxford University Press, 1994.

[14] McCarthy, P. J. Introduction to arithmetical functions. Universitext. SpringerVerlag, New York, 1986.

[15] Watkins, M. E. Connectivity of transitive graphs. J. Comb. Theory 8 (1970), 23-29. 